\title{
Kink Instability: Evolution and Energy Dissipation in Relativistic Force-free Nonrotating Jets
}

\author{
Omer Bromberg ${ }^{1}$, Chandra B. Singh ${ }^{1}$ (1) Jordy Davelaar ${ }^{2,3}$, and Alexander A. Philippov ${ }^{3,4}$ (1) \\ ${ }^{1}$ The Raymond and Beverly Sackler School of Physics and Astronomy, Tel Aviv University, Tel Aviv 69978, Israel; omerbr@tauex.tau.ac.il \\ ${ }^{2}$ Department of Astrophysics/IMAPP, Radboud University Nijmegen, P.O. Box 9010, 6500 GL Nijmegen, The Netherlands \\ ${ }^{3}$ Center for Computational Astrophysics, Flatiron Institute, 162 Fifth Avenue, New York, NY 10010, USA; sphilippov@ flatironinstitute.org \\ ${ }^{4}$ Moscow Institute of Physics and Technology, Dolgoprudny, Institutsky per. 9, Moscow region, 141700, Russia \\ Received 2019 July 9; revised 2019 August 27; accepted 2019 August 28; published 2019 October 11
}

\begin{abstract}
We study the evolution of kink instability in a force-free, nonrotating plasma column of high magnetization. The main dissipation mechanism is identified as reconnection of magnetic field lines with various intersection angles, driven by the compression of the growing kink lobes. We measure dissipation rates $d U_{B \phi} / d t \approx-0.1 U_{B \phi} / \tau$, where $\tau$ is the linear growth time of the kink instability. This value is consistent with the expansion velocity of the kink mode, which drives the reconnection. The relaxed state is close to a force-free Taylor state. We constrain the energy of that state using considerations from linear stability analysis. Our results are important for understanding magnetic field dissipation in various extreme astrophysical objects, most notably in relativistic jets. We outline the evolution of the kink instability in such jets and derive constraints on the conditions that allow for the kink instability to grow in these systems.
\end{abstract}

Unified Astronomy Thesaurus concepts: Solar coronal holes (1390); relativistic jets (1390); Plasma physics (2089); Plasma astrophysics (1261); Gamma-ray bursts (629); Magnetic fields (994); Active galactic nuclei (16)

\section{Introduction}

Relativistic jets power some of the most luminous astrophysical objects we know, like gamma-ray bursts (GRBs), microquasars, and radio-loud galaxies. It is generally accepted that the jets are launched electromagnetically, most likely by the winding of magnetic field lines that thread a rotating compact object (Blandford \& Znajek 1977; Komissarov 2001). The winding generates Poynting flux at the expense of rotational energy, which is later collimated to form a jet. Though the process of magnetic jet launching seems to be well understood, the jet physics at large distances is still a matter of active debate (e.g., see a review by Hawley et al. 2015). One of the most fundamental questions is where and how jets dissipate their magnetic energy. This has important implications on particle acceleration and emission mechanisms in the jets, the fraction of magnetic energy carried by the jets at large distances, and on the jets' stability properties.

The theory of magnetic jets stability was originally developed for magnetic confinement of plasma in tokamak facilities (e.g., Freidberg \& Haas 1973; Rosenbluth et al. 1973; Kadomtsev 1975). This theory was later applied to astrophysical jets where analytic and numerical studies were conducted in nonrelativistic (e.g., Hood \& Priest 1979; Appl 1996) as well as highly relativistic regimes (e.g., Begelman 1998; Lyubarskii 1999). In toroidal-field dominated jets, the fastest growing instability is known as kink instability. This current-driven instability (CDI) generates helical deformations in the jet, which can lead to an efficient dissipation of the jet's magnetic energy and may even disrupt the jet altogether. Linear stability analysis by Lyubarskii (1999) and by Appl et al. (2000) found the growth rates and typical wavelengths of the instability. Later Lery et al. (2000) showed that the nonlinear state is well characterized by a fastest growing mode as predicted by the linear stability analysis. The basic results of these studies, mainly the growth rates, were confirmed with numerical magnetohydrodynamic (MHD) simulations (e.g., Mizuno et al. 2009, 2012). However, a detailed numerical study of the nonlinear evolution of the instability in the relativistic regime, the relaxation condition, and most importantly, the amount and rate of the magnetic energy dissipation was not performed.

In this work we conduct a systematic study of the evolution of kink instability in highly magnetized, force-free columns, using relativistic MHD simulations. We start by summarizing the linear theory of kink instability in various magnetic field configurations in Section 2. We then describe the nonlinear evolution of the kink mode and outline the predictions for the magnetic relaxation, which were established in the lowmagnetization regime (Section 3). In Section 4 we discuss the minimal energy state and how it can be used to predict the amount of dissipated energy. In Section 5 we outline the numerical setup, and in Section 6 we report our findings. We identify the dissipation mechanism, verify the relaxation criterion and quantify the amount of energy dissipation that takes place in the process. We discuss the implications for astrophysical jets and twisted magnetic loops (Section 7) and conclude in Section 8.

\section{Kink Instability Linear Evolution}

CDI modes tend to grow on resonant surfaces that satisfy the condition $\boldsymbol{k} \cdot \boldsymbol{B}=0$, where $\boldsymbol{k}$ is the wave vector of the growing mode, and $\boldsymbol{B}$ is the vector of the local magnetic field (Rosenbluth et al. 1973; Kadomtsev 1975). In cylindrical geometry this translates to the condition $k B_{z}+(m / r) B_{\phi}=0$, with $k, m$ being the wave numbers in the longitudinal and azimuthal directions respectively, and we use standard cylindrical coordinates $(r, \phi, z)$. In a periodic box, the vertical wave number can be expressed as $k=2 \pi n / L$, where $n$ is an integer number and $L$ is the longitudinal box size. The resonant 
condition can also be written as

$$
k P+m=0,
$$

where $P \equiv r B_{z} / B_{\phi}$ is the pitch of the magnetic field. Linear stability analysis for jets of finite length show that resonant modes grow on discrete surfaces that fulfill the condition $k_{\text {res }} \simeq-m / P_{0}$, where $P_{0}$ is the pitch at the axis. ${ }^{5}$ The fastest growing mode is the $m=-1$ mode, known as the kink mode. In practice, it grows over a range of wavelengths, where the maximum growth rate occurs at a wave number

$$
k_{\max } \simeq 0.745 \times 1 / P_{0},
$$

having a growth rate

$$
\Lambda_{\max }=0.133 v_{\mathrm{A}} / P_{0},
$$

where $v_{\mathrm{A}}$ is the Alfvén velocity. These scalings are almost independent of the pitch profile (Appl et al. 2000).

\section{Nonlinear Evolution and Relaxation}

Though the linear growth of the kink mode has a rather weak dependence on the pitch profile, its evolution in the nonlinear regime changes with the pitch profile. A theoretical understanding of the nonlinear regime and of the relaxation process was obtained for nonrelativistic configurations (Kadomtsev 1975). The plasma in this case has low magnetization, $\sigma=$ $B^{2} / 4 \pi \rho c^{2} \ll 1$, and it resides in a periodic box with length $L=2 \pi P_{0}$ along the jet axis, which only allows for the $n=1$ mode to grow.

1. In configurations where $P(r)$ increases with $r$ (increasing pitch, IP) there is a resonant surface that corresponds to the fastest growing mode, $P\left(r_{\text {res }}\right)=1 / k_{\max } \approx 4 P_{0} / 3$. The mode's wavelength can be expressed as

$$
\lambda_{\mathrm{res}}=\frac{2 \pi}{k_{\mathrm{res}}} \simeq \frac{8 \pi}{3} P_{0}
$$

It generates a helical twist in the jet, which grows inwards to the resonant surface, and leads to the formation of a largescale current sheet at a radius $\sim r_{\text {res }}$. As the mode continues to grow, the current sheet extends to the regions between the kink lobes, gets compressed, and eventually breaks due to resistive instabilities (Kadomtsev 1975). The dissipation may proceed in a more stochastic fashion, through smallscale current sheets or turbulence, while maintaining the global helical shape of the kink mode.

Since in this case the kink mode perturbs only the jet inside of $\sim r_{\text {res }}$, it is termed an internal kink mode. If the resonant surface is located outside the boundary of the current-carrying core (e.g., the jet boundary), the mode will spread out until it engulfs the entire core, generating a global helical structure. We term this mode an external kink mode. ${ }^{6}$

2. In configurations where the pitch profile decreases with radius (decreasing pitch, DP), there is no surface fulfilling the resonance condition in Equation (2). Any large-scale kink mode that grows is expected to break apart, avoiding

\footnotetext{
5 In a magnetic configuration of a uniform pitch, $P_{0}$ is equal to the radius of jet core, which carries most of the current that supports the toroidal-magneticfield component.

6 In plasma physics literature a kink mode is called external if it grows on the plasma-vacuum boundary, which is absent in astrophysical systems. Instead, we term a kink mode that grows on, or outside the boundary of the currentcarrying core, and deforms its shape as an external mode.
}

the formation of a prominent global current sheet. The result is a more stochastic evolution, likely without a large-scale helical pattern. As the global mode continues to grow, it may eventually disrupt the entire jet. Magnetic configurations with the DP profile are naturally less stable, so it remains questionable if and how these configurations can be realized in the first place.

3. The case of a constant pitch can be considered as a special case. Since the fastest growing mode corresponds to a pitch value $P\left(r_{\text {res }}\right)=1 / k_{\max }>1 / P_{0}$, there is no resonant surface. In the limit of a small pitch $\left(P_{0} \ll L\right.$, $R_{j}$ ) the evolution will be similar to the DP case. A nonresonant mode will grow at $r \simeq P_{0}$, and will spread outward leading to a global/stochastic dissipation. In the limit of a large pitch, the large value of $P_{0}$ stabilizes it against the growth of internal modes. Moreover, the kink growth rate quickly decreases with growing $P_{0}$, thus, the jet becomes stable for further dissipation by the kink instability (Appl et al. 2000).

The dissipation of electromagnetic (EM) energy takes place mostly during the nonlinear stage through reconnection and stochastic/turbulent dissipation. Although the magnetic configuration changes during the dissipation process, the total helicity is roughly conserved (e.g., Taylor 1974, 1986, 2000). The magnetic field configuration gradually relaxes into a minimal energy state, known as a Taylor state (Taylor 1974), which maintains

$$
\boldsymbol{j}(r)=\alpha \boldsymbol{B}(r),
$$

where $\alpha$ is constant and it can be expressed as

$$
\alpha=\frac{\boldsymbol{B} \cdot(\boldsymbol{\nabla} \times \boldsymbol{B})}{B^{2}} .
$$

Note that Equation (5) corresponds to a force-free state, since $\boldsymbol{j} \times \boldsymbol{B}=0$. A magnetic field configuration that is both cylindrically symmetric and obeys condition (5) can be expressed as

$$
\begin{aligned}
& B_{z}=B_{0} J_{0}(r \alpha) \\
& B_{\phi}=B_{0} J_{1}(r \alpha),
\end{aligned}
$$

where $J_{0}$ and $J_{1}$ are the zeroth and first Bessel functions of the first kind. This configuration is unstable for $m=-1$ kink modes that satisfy (Voslamber \& Callebaut 1962)

$$
k<0.272 \alpha \text {. }
$$

In a periodic box of length $L$ the minimal $k$ that can be excited is $k=\pi / L$, corresponding to a wavelength $\lambda=2 L$. If $\pi /$ $L>0.272 \alpha$, the configuration is stable to kink. Namely, for a given box size configurations with $\alpha \lesssim 4 \pi / L$ are stable for kinking. In this case, the value of the pitch on the axis is

$$
P_{0}=\frac{2}{\alpha} \gtrsim \frac{L}{2 \pi},
$$

which is just the Kruskal-Shafranov (K-S) criterion (Shafranov 1956; Kruskal \& Tuck 1958) for the stabilization of kink instability. Jets with a Bessel profile and a small aspect ratio ("infinitely long jets") are stable for all kink modes if they 
satisfy

$$
\alpha R_{j} \leqslant 3.176
$$

where $R_{j}$ is the cylindrical jet radius (Voslamber \& Callebaut 1962). For values of $\alpha R_{j}$ in the range $3.176 \leqslant \alpha R_{j} \leqslant 3.832$ the jets become increasingly unstable and for $\alpha R_{j}>3.832$ they are unstable for all modes with $k<0.272 \alpha$. It is important to note that for $\alpha R_{j} \leqslant 3.832$ the first zero of $J_{0}$ is located inside $R_{j}$, and the first zero of $J_{1}$ falls outside $R_{j}$. This implies that $B_{z}$ flips sign in the outer part of the jet, while $B_{\varphi}$ maintains its direction. We find evidence for such a behavior in several configurations that we tested in this work. All of them reached a condition of marginal stability with $\alpha R_{j}$ being close to the value given by Equation (10).

\section{Minimal Energy State}

If the final configuration is fully relaxed, the magnetic field profile can be described by the set of Bessel functions given in Equation (7). Three parameters are required to calculate the final EM energy in this case: $B_{0}, \alpha$, and $R_{j}$. The dissipation process conserves two quantities to a good accuracy: the total helicity and the total axial magnetic flux. A third condition can come comes from constraining the final $\alpha$, (e.g., by the stability criterion given in Equation (10)), or the radius of the dissipated region.

The total helicity in a volume is defined by

$$
H \equiv \int_{V} \boldsymbol{A} \cdot \boldsymbol{B} d V
$$

As such, it is a gauge-dependent quantity. Gauge invariance is possible in specific magnetic field typologies, for example, when $\boldsymbol{B}$ is tangent to the boundary of the volume, and its evolution conserves longitudinal magnetic flux (Browning et al. 2008). The situation of an axisymmetric magnetic field with a vanishing radial component on the boundary is ideal for the helicity conservation. In this case the helicity can be described as

$$
H=2 \pi L\left[2 \int_{0}^{R} \frac{\Psi\left(r^{\prime}\right)}{2 \pi} \frac{2 I\left(r^{\prime}\right)}{r^{\prime}} d r^{\prime}+\left.\left(A_{z}(r) \frac{\Psi(r)}{2 \pi}\right)\right|_{0} ^{R}\right],
$$

where $\Psi(r)$ is the magnetic flux within radius $r$, and $I(r)$ is the current within the same radius. Taking a gauge $A_{z}(R)=0$, the second term vanishes, and we are left with the first term, which we define as

$$
K(R) \equiv 2 \int_{0}^{R} \frac{\Psi\left(r^{\prime}\right)}{2 \pi} \frac{2 I\left(r^{\prime}\right)}{r^{\prime}} d r^{\prime}
$$

$K\left(R_{j}\right)$ is largely conserved throughout the evolution of the system. If the magnetic configuration in the final state can be approximated as a cylindrically symmetric Taylor state, $K$ and $\Psi$ can be expressed as (see Appendix A)

$$
\begin{gathered}
K=\frac{B_{0}^{2}}{\alpha^{2}} \Upsilon\left(R_{j}\right), \\
\Psi=2 \pi B_{0} \int_{0}^{R_{j}} J_{0}(\alpha r) r d r,
\end{gathered}
$$

with

$$
\Upsilon\left(R_{j}\right)=\int_{0}^{\alpha R_{j}}\left[J_{0}(\xi)^{2}+J_{1}(\xi)^{2}\right] d \xi-J_{0}\left(\alpha R_{j}\right) J_{1}\left(\alpha R_{j}\right) R_{j} .
$$

Substituting the initial $K$ and $\Psi$ values in Equations (14) and (15), and adding a constraint on the relaxed configuration, for example, Equation (10), gives a closed set of equations from which we can estimate the energy in the final state.

Though the outlined theory of magnetic relaxation has been applied in the nonrelativistic regime applicable for solar flare (Browning et al. 2008), it is unclear whether the same theory applies to relativistic plasma in extreme astrophysical environments of relativistic jets or twisted magnetic loops in the accretion disk coronae. First, since $\sigma \gg 1$, the dissipation process generates thermal pressure, which can be of the order of the mass energy density of the plasma, and it is unclear whether a force-free condition can be sustained. Second, it is unclear which process, turbulence or reconnection, dominates the dissipation. We employ numerical simulations to test the nonlinear evolution of kink instability in relativistic plasma and compare the results to the expectations from the nonrelativistic theory.

\section{Numerical Setup}

For our studies of the kink instability we use the software PLUTO (Mignone et al. 2007, 2012), a three-dimensional relativistic MHD code designed to simulate astrophysical flows with high Mach numbers and moderate to high values of the magnetization parameter. (e.g., Mignone et al. 2010, 2013; Bodo et al. 2013). PLUTO has a very flexible numerical scheme, which allows testing of how the details of the implementation affect the results. Our chosen scheme consists of a third order Runge-Kutta time stepping, piecewise parabolic reconstruction with a harmonic limiter, HLL Riemann solver, and we use a Courant number of 0.3 . In the case of high $\sigma$, low plasma $\beta$ regime more accurate solvers like HLLD can lead to numerical problems (Mignone et al. 2007; Anjiri et al. 2014). In order to avoid unphysical states, slopelimited reconstruction with the MinMod limiter is adopted to handle shocks, and we use constrained transport to enforce div $\boldsymbol{B}=0$. We use an ideal equation of state with an adiabatic index $4 / 3$.

To examine the evolution of internal kink modes we set the computational box inside the jet, so that the jet boundaries lie outside the box. Our study is focused on relativistic jets, however, the approximations we make are also relevant for other systems, such as twisted magnetic loops in the accretion disk coronae. We therefore set up a second configuration where a high $\sigma$ core (the "loop") is embedded in a magnetized external medium, which is relevant for such a case (e.g., Gordovskyy \& Browning 2011). In such configuration we examine the evolution of external kink. We use a Cartesian grid with periodic boundaries in the direction of the jet axis, $z$, and outflow boundary conditions in the transverse, $x-y$, directions.

We perform simulations in a reference frame comoving with the jet. In the coronal configuration, this setup corresponds to the frame of the magnetic loop. For simplicity we neglect gradients in the longitudinal velocity and rotation (Mizuno et al. 2009). Even though the magnetic field in the jet is predominantly toroidal in the lab frame, the poloidal field cannot in general be neglected because one has to compare the 
fields in the comoving frame where the toroidal field is much lower. For example, in equilibrium configuration with cylindrical symmetry, the poloidal and toroidal fields in the comoving frame are comparable (Lyubarsky 2009). Therefore, analysis of kink instability for astrophysical jets has to take into account the non-negligible poloidal field. In addition, since it is very likely that before the flow becomes kink unstable the plasma is cold, force-free configuration is a good initial condition. In the absence of rotation ${ }^{7}$ the hoop stress has to be balanced by the gradient in the poloidal magnetic pressure, so an equilibrium configuration generally has a core of poloidal field near the axis.

We set up a helical magnetic field with a nonrotating, forcefree configuration (Mizuno et al. 2009):

$$
\begin{aligned}
& B_{\mathrm{r}}(r)=0, \\
& B_{\mathrm{z}}(r)=\frac{B_{0}}{\left[1+(r / a)^{2}\right]^{\zeta}}, \\
& B_{\phi}(r)=\frac{a B_{z}}{r} \sqrt{\frac{\left[1+(r / a)^{2}\right]^{2 \zeta}-1-2 \zeta(r / a)^{2}}{2 \zeta-1}} .
\end{aligned}
$$

This profile has a monotonic pitch determined by the parameter $\zeta$. The pitch is increasing for $\zeta<1$ and decreasing if $\zeta>1$. The radius of the $B_{z}$-dominated core is of the order of the value of pitch at the axis, $P_{0}=a \sqrt{1 / \zeta}$. We consider two values of $\zeta=0.64,1.44$ representing configurations of IP and DP with $P_{0}=1.25 a, 5 / 6 a$, respectively. A third configuration we study is based on Bodo et al. (2013), which is also a force-free and static configuration. In this case the helical core is embedded in a uniform axial "external" field. Such configuration can be applicable for twisted magnetic field loops in accretion disk coronae or in magnetospheres of magnetars (Beloborodov 2009; Parfrey et al. 2013). The field configuration has the form

$$
\begin{aligned}
& B_{\phi}=\frac{B_{0} R}{r} \sqrt{1-e^{-\frac{r^{4}}{a^{4}}}}, \\
& B_{z}=\frac{B_{0} R P_{0}}{a^{2}} \sqrt{1-\sqrt{\pi}\left(\frac{a^{2}}{P_{0}^{2}}\right) \operatorname{erf}\left(\frac{r^{2}}{a^{2}}\right)},
\end{aligned}
$$

where $R$ is the cylindrical radius of the computational box, and $P_{0}$ is the value of the pitch on the axis. In this work we study the kink evolution in the case of initial high magnetization at the axis, defined as $\sigma \equiv b^{2} / 4 \pi \rho c^{2}$. We perform simulations with a peak magnetization $\sigma=10$, and set a uniform pressure and mass density in the box as an initial condition. We normalize all length scales by $a$, time units by $a / c$, energy density by $\rho c^{2}$, and strength of the magnetic field by $\sqrt{4 \pi \rho_{0} c^{2}}$. In these units the values of the gas density and pressure are $\rho=1$ and $p=0.01$ respectively. The magnetic fields and related pitch profiles used in this work are presented in Figure 1. To initiate the kink instability we introduce random perturbations to the radial velocity $v_{r}=\eta_{N} \delta v e^{-r / 2 a}$, where

\footnotetext{
In the presence of rotation, the hoop stress can be entirely compensated by the electric force. In this case cylindrically symmetric configuration is known to be stable to kink instability (Istomin \& Pariev 1996; Lyubarskii 1999). However, if the profile of the poloidal field shows substantial transverse gradient, growth of the instability in rotating and nonrotating equilibria is qualitatively similar (Sobacchi et al. 2017). A numerical investigation of this case will be performed in a separate work.
}

$\delta v=0.1 c$ and $\eta_{N}$ is a random number drawn from a uniform distribution in the range $\{-1,1\}$. We also performed simulations with $\delta v=0.01 c$ and found no difference in the linear growth rates and the nonlinear evolution.

We set the size of the box in the longitudinal direction so that it fits several kink wavelengths $\left(L \simeq 2 \pi n / k_{\max }, n>1\right)$. This allows us to test the effect of interactions of multiple modes on the dissipation process. To study the dependence of the dissipation on the number of excited modes, we vary the size of the computational box, thus allowing for different numbers of kink wavelengths to grow. Table 1 summarizes the magnetic field profiles and box sizes we used. To sample the dissipation scales properly in the MHD simulations we need to resolve the core with at least 15 cells per unit radius $a$. A convergence tests with 30 and 45 cells per unit radius showed no significant difference in the evolution of the kink instability. The convergence tests are presented in Appendix B.

\section{Results}

\subsection{Overall Structure and Growth Rates}

The evolution of the kink instability can be characterized by several stages, as depicted in Figures 2-4. The figures show a series of snapshots from various evolutionary stages of the studied systems. We show results for the large box runs, cases $\mathrm{IP}_{\mathrm{b}}$ (top), $\mathrm{DP}_{\mathrm{b}}$ (middle), and $\mathrm{CO}_{\mathrm{b}}$ (bottom). Figures 2 and 4 show the current density in the $z$ direction and Figure 3 shows the thermal pressure at the same times. The growth rates of the kink modes in the three cases can be seen in Figure 5, which shows the average value of $E^{2}$ in the box normalized by the initial value. The evolution in all cases is characterized by an initial fast exponential rise, evident as a linear growth in Figure 5 and demonstrated in the left-most panels of Figures 2-4. The growth rates in this stage match the analytic predictions of the linear theory quite well, as can be seen in the zoomed-in box of Figure 5.

Beyond the linear stage, the evolution depends on the magnetic field configuration, in particular on the pitch profile. In the IP case the kink mode grows on a resonant surface. Since the mode is mostly confined to that surface it grows faster in the longitudinal direction, increasing the width of the individual kink lobes until they touch each other (see Figure 2 at $t=200[a / c])$. At this point the exponential growth saturates and the kink mode starts to "inverse cascade" through a series of coalescence events (mergers), where in each merger the longitudinal wave number, $n$, is reduced by unity. This phase is seen in Figure 5 as a series of bumps in the value of $E^{2}$. As the inverse cascade continues the longitudinal wave vector, $k$ decreases until it reaches $k_{\min }=\frac{\pi}{L}$, corresponding to an $n=1 / 2$ wave number, and the merger stops (the right-most images in Figures 2-4). The merger process breaks the ordered structure of the magnetic field, forming a stochastic turbulent configuration, which slowly relax to a minimal energy state once the mergers ends (after $\sim t=400[a / c]$ ).

In the DP case, there is no resonant surface. The kink mode grows in amplitude as well as in width, until the kink lobes touch each other and begin to merge. Here we identify a major merging episode, which brings the wave number down to a low $n$ value in a single event (as oppose to the gradual merging process in the IP case). It is followed by secondary, weaker events, which destroy the structure of the kink mode completely. As in the IP case, the merger process breaks the 

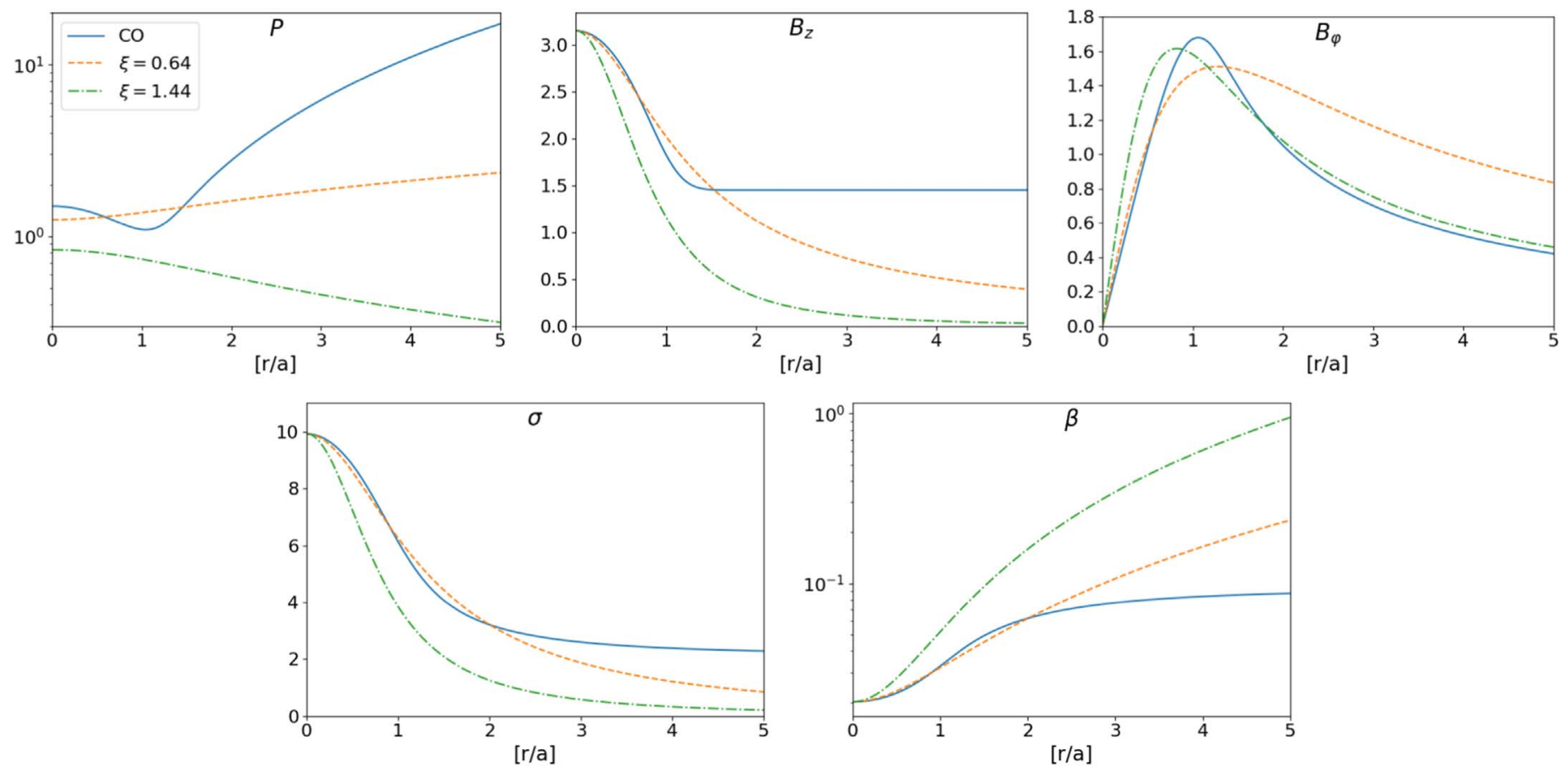

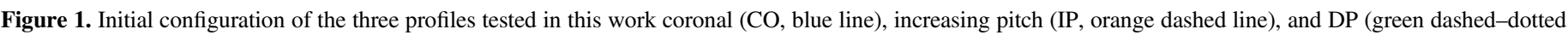
line). Panels show from left to right, top to bottom: Pitch (in $\log$ scale), $B_{z}, B_{\varphi}, \sigma$ and plasma $\beta$ (in log scale).

Table 1

Simulations Parameters

\begin{tabular}{lcccc}
\hline \hline Name & $\sigma_{0}$ & $\begin{array}{c}\text { Box } \\
\text { Dimension }\end{array}$ & Resolution & $\begin{array}{c}t_{f} \\
{[a / c]}\end{array}$ \\
\hline $\mathrm{IP}_{\mathrm{a}}$ & 10 & $80 \times 80 \times 20$ & $1200 \times 1200 \times 300$ & 2000 \\
$\mathrm{IP}_{\mathrm{b}}$ & 10 & $120 \times 120 \times 40$ & $1800 \times 1800 \times 600$ & 3000 \\
\hline $\mathrm{DP}_{\mathrm{a}}$ & 10 & $80 \times 80 \times 14$ & $1200 \times 1200 \times 210$ & 1760 \\
$\mathrm{DP}_{\mathrm{b}}$ & 10 & $100 \times 100 \times 28$ & $1500 \times 1500 \times 420$ & 1760 \\
\hline $\mathrm{CO}_{\mathrm{a}}$ & 10 & $30 \times 30 \times 20$ & $450 \times 450 \times 300$ & 2000 \\
$\mathrm{CO}_{\mathrm{b}}$ & 10 & $30 \times 30 \times 80$ & $450 \times 450 \times 300$ & 2000 \\
\hline
\end{tabular}

global structure of the magnetic field, forming a stochastic turbulent structure, which eventually relaxes to a stable configuration.

In the $\mathrm{CO}$ case the resonant surface is located very close to the edge of the helical core. As a result, the kink mode grows close to the core edge and quickly becomes an external mode to the core. The high magnetic tension of the external longitudinal field prevents the kink mode on the core boundary from growing to a large amplitude with respect to the core's crosssectional radius. Instead, the growth takes place mostly along the boundary, more extremely than in the IP case, creating a current sheet at edge of the core, which quickly breaks down. As the instability continues to grow, the kink mode inverse cascades to longer wavelength via a series of mergers that ends at $600 \lesssim t \lesssim 800[a / c]$ when it reaches the smallest $k$ allowed in the box. It results in a mildly perturbed core with a stochastic structure of magnetic fields, which slowly relax to the minimal energy state. During the evolution of the kink instability, the radius of the dissipated core grows. As the core pushes against the magnetic field in the medium, external matter mixes into the core through instabilities at the boundary.

\subsection{Energy Dissipation}

The dissipation of the EM energy occurs mostly in current sheets and is tightly related to the evolution of the kink instability. The current sheets are evident in Figure 2 as local extrema in the current density with filamentary shape. Figure 3 shows the corresponding thermal pressure measured at the same time. The pressure peaks match the location of the filaments of $J_{z}$, indicating that most of the dissipation occurs in the current sheets.

During the linear stage a global current sheet is formed at the edge of the kink mode, in regions where the magnetic field is compressed by the growing amplitude of the mode. Since the volume of the current sheet is small and the magnetic field at the location of the sheet is weak, the dissipated energy is minute. Figure 6 depicts the value of the EM energy at different times in the three configurations. The initial slow decline in the EM energy evident in all panels marks the dissipation during the linear stage.

The linear stage ends when the individual kink lobes touch each other and begin to merge. As a result, the current sheet, which was confined to the outer edge of the kink mode, extends inwards along the surface of contact between the kink lobes and becomes prominent (Figures 2 and 4 at $t=200[a / c]$ ). The dissipation process in the current sheet can be attributed to reconnection of magnetic field lines with varying intersection angles, which is driven by the compression of the merging kink lobes. In the IP and CO profiles the reconnection angle is rather small, while in the DP case the reconnecting field lines are close to be antiparallel. Thus, in the DP case the dissipation rate is faster and the kink evolution differs from the first two cases.

As the merging process progresses, the helical current sheet becomes increasingly thinner until it eventually breaks down to small structures, due to resistive effects. The substructures further break into smaller structures, resulting in a turbulent configuration of the magnetic field. It gradually fills the entire 

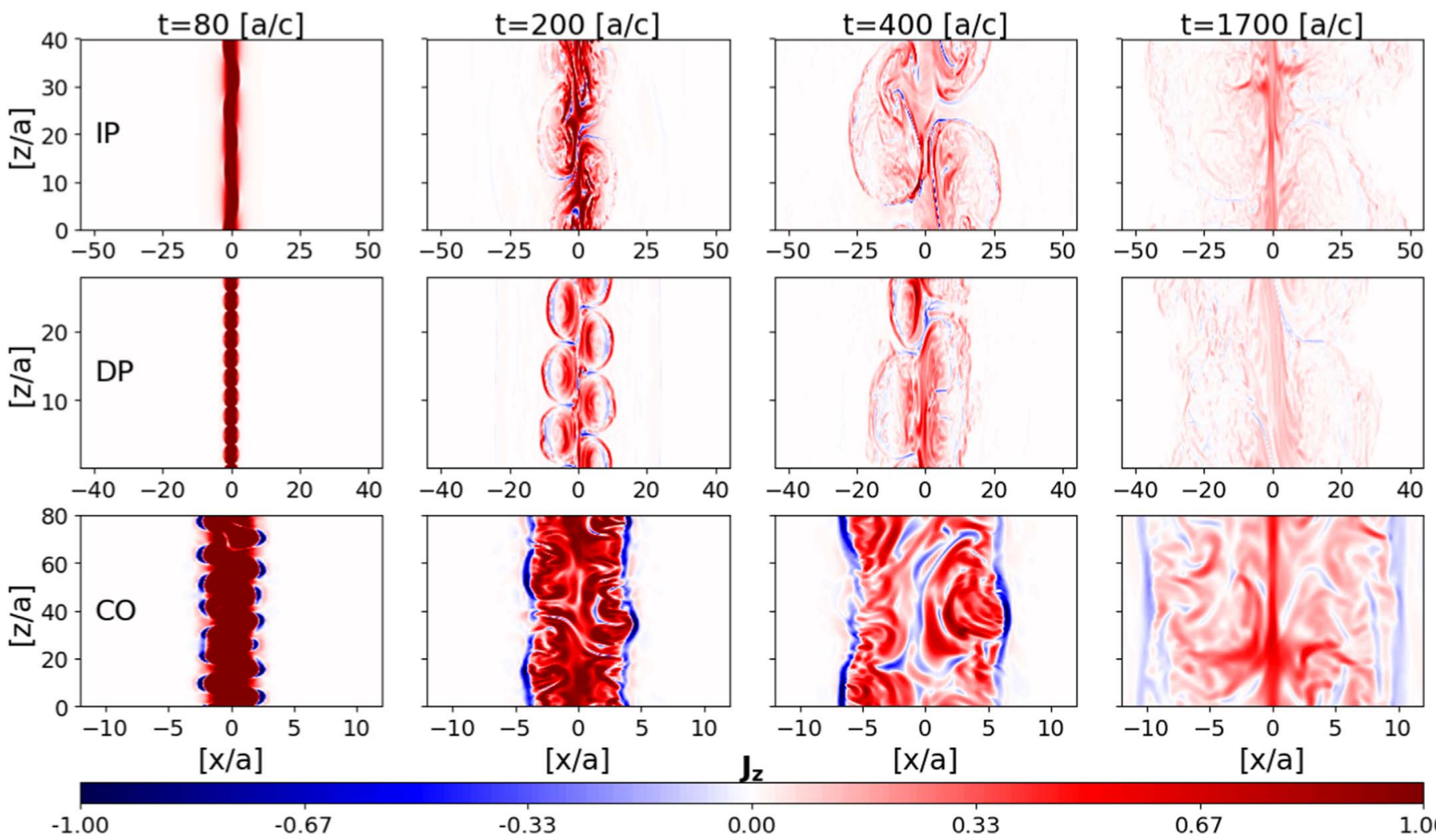

[x/a]

[x/a]

$$
-1.00
$$

$-0.67$

$-0.33$

0.33

0.67

1.00

Figure 2. Evolution of the kink instability in cases $\mathrm{IP}_{\mathrm{b}}, \mathrm{DP}_{\mathrm{b}}$, and $\mathrm{CO}_{\mathrm{b}}$. Shown are values of $J_{z}$ on the $x-z$ plane. Current sheets are seen as peaked color filaments.

volume inwards to the current sheet and contributes to the dissipation. The energy that is driven into the current sheet through the mergers of the kink lobes, cascades down to the small-scale turbulence, keeping the dissipation rate high.

Once the merging stops, energy is no longer pumped into the turbulence and the dissipation rate is reduced. This transition is manifested in Figure 6 as a break in the dissipation rate evident in all panels, occurring at times consistent with the end of the merger episodes. In the IP and CO cases the mergers reduce the wave number of the kink mode progressively, until it reaches the minimal value allowed in the box, $n=1 / 2$. Therefore, the duration of the kink mode inverse cascade depends on the longitudinal size of the box, as more waves are excited in larger boxes. Indeed, as can be seen in Figure 6 the transitions from the fast, merger-driven dissipation to the slower, turbulence dissipation occur at later times in the large boxes. In the DP case, the merger is instantaneous and its duration is independent of the box size. The large angles between the reconnecting field lines result in pumping more energy into the current sheets than in the IP and CO cases. This is evident in the higher spikeof the electric field seen in Figure 5. As a result, the dissipation rate in the DP case is higher and the total fraction of dissipated EM energy is larger as well (see below).

\subsection{Relaxation}

The high dissipation rate continues as long as fresh energy is pumped into the turbulence by the inverse cascade of the kink mode. Once the K-S condition (Equation (9)) is met, the kink instability relaxes and energy transfer to the turbulence stops. The turbulence continues to dissipate the energy contained in them at a slower rate until the system reaches a minimal energy state. The magnetic energy configuration at this point is close to a Taylor state, characterized by a relatively flat $\alpha$ profile (Equation (6)). During the dissipation process the pressure profile steepens and the pressure gradient becomes of the order of of $\nabla B^{2} / 8 \pi$. As the configuration approaches the relaxed state, the pressure profile flattens again and the plasma becomes force-free, ${ }^{8}$ as required by the ideal Taylor state (see Equation (5)). Figure 7 shows the radial distribution of the EM energy density, $e_{\mathrm{EM}}=\frac{1}{8 \pi}\left(E^{2}+B^{2}\right)$, together with the distribution of the thermal energy density, $u=T_{00}-\rho \Gamma^{2} c^{2}$, averaged over $z$ and $\varphi$. In our case Lorentz factors are small and $u \simeq 3 p$. It can be seen that although the ratio of EM to thermal energy density varies substantially between the three cases, the final pressure profile is flat and the configuration is force-free.

Figure 8 shows the radial profile of $\alpha$ averaged over $z$ and $\varphi$, for all magnetic field profiles and box sizes discussed in this work. Shown are the initial values (in dashed line) and the values at the end of the simulations. In all large box simulations the $\alpha$ at the core is lower than in the corresponding simulations of small boxes, and its profile across the box is flatter. This likely occurs since in the large boxes the kink mode initially has a higher wave number, which takes longer to inverse cascade to the lowest $n$. As a result the magnetic field distribution has more time to dissipate energy efficiently and thus it can reach a lower energy state.

The magnetic field configuration relaxes into a Taylor state, which can be represented by two Bessel functions of the first kind (see Equation (7)) with the first zero of $J_{0}$ falling inside the dissipated region, implying a reversal of $B_{z}$ close to $R_{j}$. Figure 9

\footnotetext{
8 In the absence of rotation the transverse force balance equation is $\nabla p+\boldsymbol{J} \times \boldsymbol{B}=0$. A flat radial pressure profile implies that $(\boldsymbol{J} \times \boldsymbol{B})_{r} \simeq 0$, thus the plasma is at a force-free state in the transverse direction.
} 

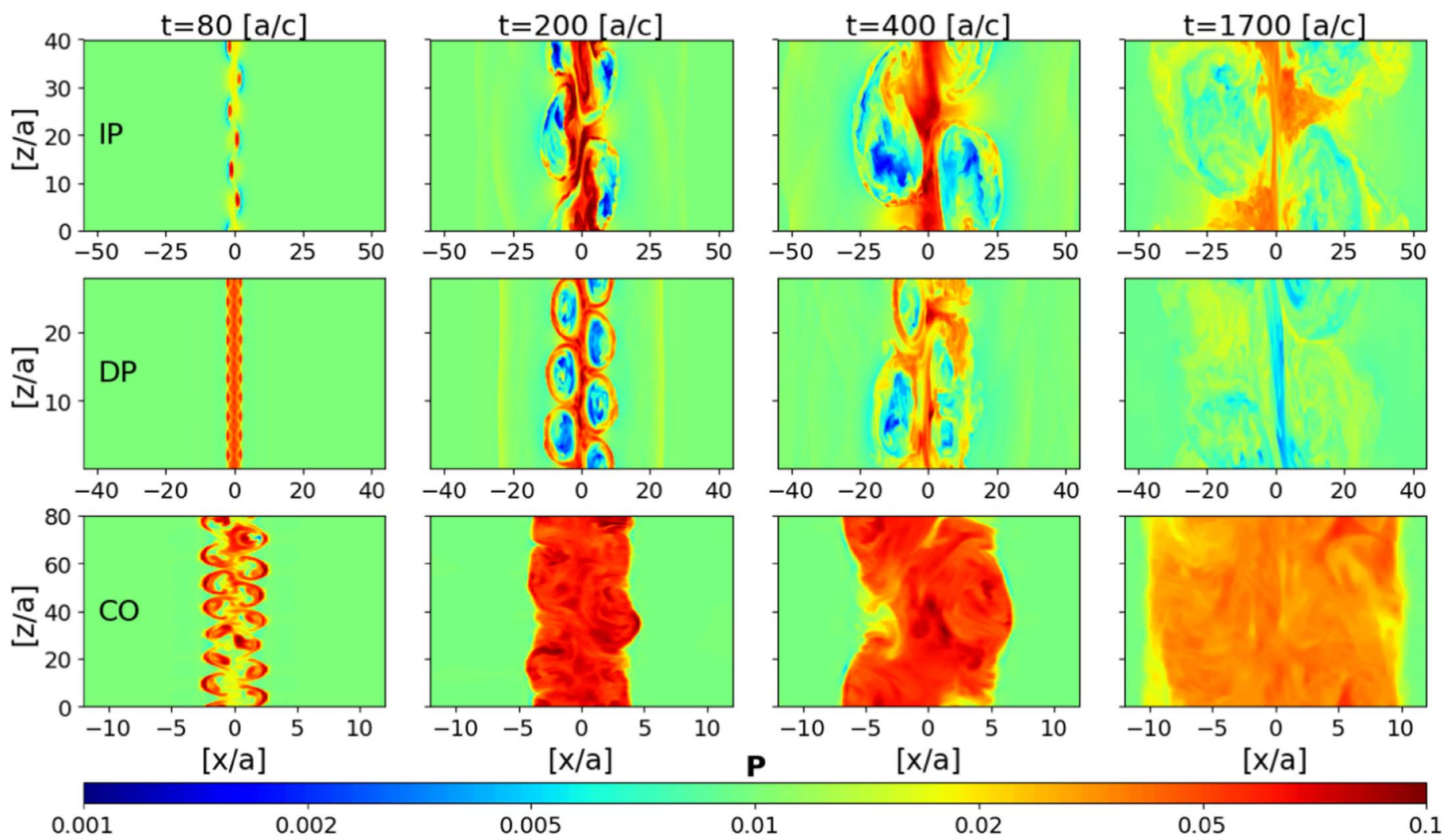

0.002

0.005

0.01

0.02

0.05

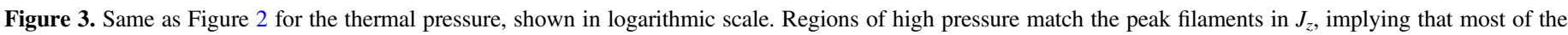
dissipation is occurring in current sheets. The pressure in the right-most column is in the course of becoming evenly distributed across the dissipated region.

shows the magnetic field profiles at the end of runs $\mathrm{IP}_{\mathrm{b}}, \mathrm{DP}_{\mathrm{b}}$, and $\mathrm{CO}_{\mathrm{b}}$ averaged over $z$ and $\varphi$. We plot in dotted lines the best fits of the Bessel functions to the configurations. The black dashed line shows $R_{j}$. It can be seen that in all three cases, the distribution at the central core fits a Taylor profile with the same normalization applied for $B_{z}$ and $B_{\varphi}$. At the outer parts of the dissipated cylinder, the reversal of $B_{z}$ required by the relaxation criterion is less evident in the IP and DP profiles. In the IP case this is partly due to the averaging over the azimuthal direction, which washes out the indications of a reversed field. To demonstrate that we show in Figure 10 the value of $B_{z}$ in a cross-sectional cut at the $x-y$ plane, in the middle of the box, The red dashed line marks $R_{j}$ in each configuration. A reversal of the vertical field component is evident in both the IP and the DP cases. No field reversal is seen in the coronal case.

In the $\mathrm{CO}$ case the strong magnetic field in the medium, prevents the kink mode from growing to large amplitude with respect to the core cross section, before it breaks down to small-scale turbulence. Nevertheless, mode merging still occurs in the core, as seen in Figure 4, and it likely serves as the energy source for the turbulent dissipation as in the other cases. The small amplitude of the kink mode prevents the flip in the direction of $B_{z}$ from occurring at the outer core part, which is important for obtaining the zero-point in $J_{0}$ seen in the IP and the DP cases. As a result the magnetic field relaxes into a Taylor state with a small $\alpha$, which corresponds to Bessel functions with zero-points outside of $R_{j}$. The best-fitted $\alpha$ values for the three magnetic field configurations in the large boxes are $\alpha=0.18,0.12$, and $0.07[1 / a]$ for the DP, IP, and coronal profiles, respectively.

\subsection{Final Energy and the Minimal Energy State}

We find that the dissipation process conserves the total magnetic flux up to $R_{j}$ and the total helicity with zero gauge, $K$ (Equation (13)), within $\sim 10 \%$ in all configurations. A similar fraction of the magnetic energy leaks out through the boundary during the simulation and is likely causing the drop of $K$. Thus, Equations (14) and (15) can be used to evaluate the final energy in the box, assuming the system has relaxed to an axially symmetric Taylor state. To close the equations we take $R_{j}$ at the end of each simulation and calculate the values of $\alpha$ and $B_{0}$ of the corresponding Taylor state. We then compare the EM energy of the Taylor state to the actual EM energy in the box and evaluate how close the system is to a minimal energy. For consistency, we compare the $\alpha R_{j}$ of the obtained Bessel functions to the theoretical value of a minimal energy state derived from the linear stability analysis (Section 4).

Figure 11 shows the total EM energy as a function of $r$ at $t_{0}$ (solid blue line) and $t_{f}$ (orange dashed line) for runs $\mathrm{IP}_{\mathrm{b}}$ and $\mathrm{DP}_{\mathrm{b}}$. We plot in a green dotted-dashed line the energy distribution of a Taylor state (Equation (7)) with $B_{0}$ and $\alpha$ obtained from conservation of $\Psi\left(R_{j}\right)$ and $K\left(R_{j}\right)$ (see Appendix A). The black vertical line shows $R_{j}$ at each configuration. In the IP case, about $50 \%$ of the initial energy is estimated to be available for dissipation. By the end of the simulation $40 \%$ of the initial energy has been dissipated, suggesting that the system is close to a minimal energy state. In addition, the obtained $\alpha R_{j}$ of the Taylor state is very close to the theoretical stability value $\alpha R_{j}=3.176$ (Voslamber \& Callebaut 1962). In the DP case $60 \%$ of the total EM energy was dissipated by the end of the simulation, where $\sim 75 \%$ of the total energy up to $R_{j}$ is estimated to be available for 

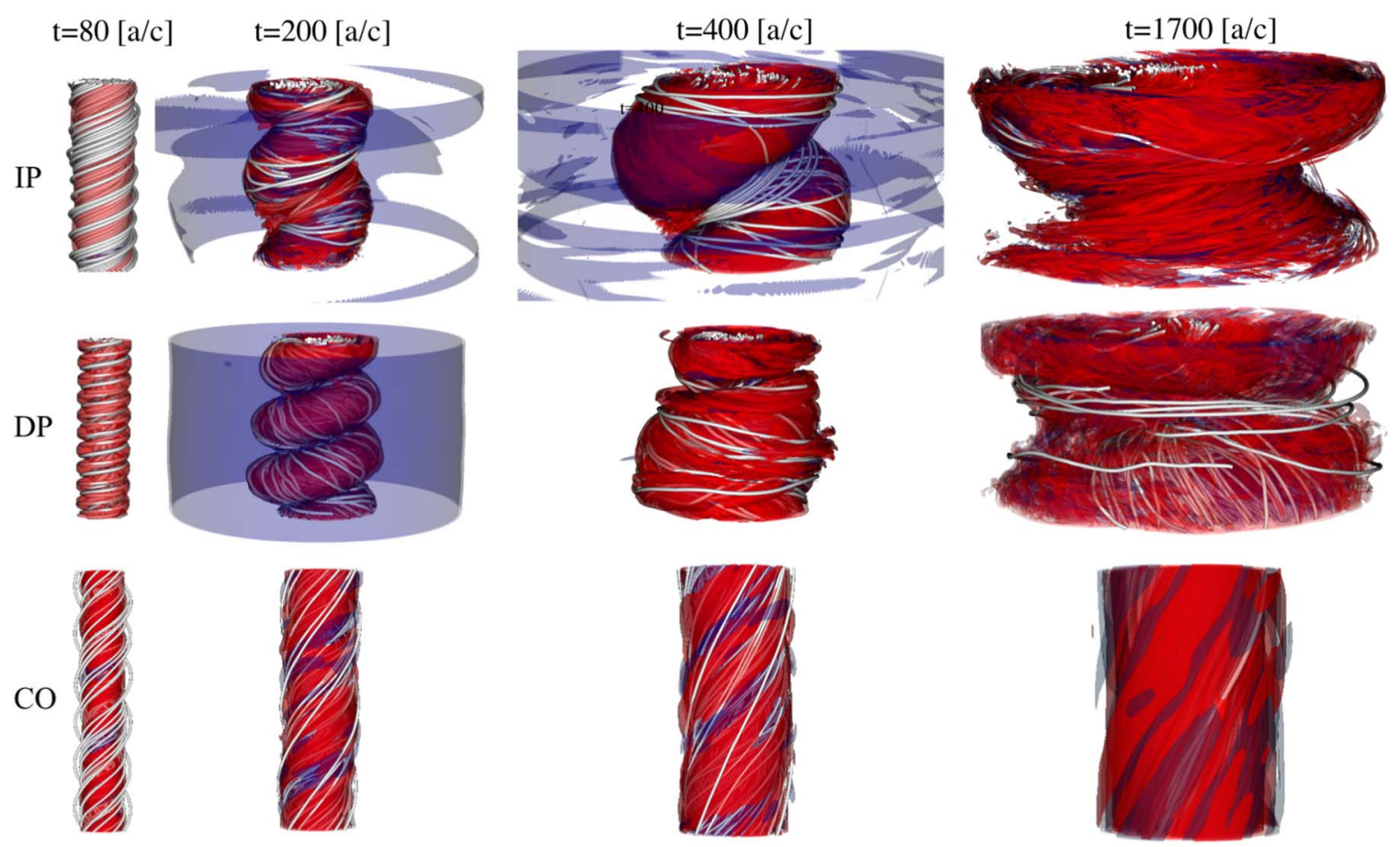

Figure 4. 3D color rendering of $J_{z}$ at the same times and color range as in Figure 2. Magnetic field lines are shown as white tubes.

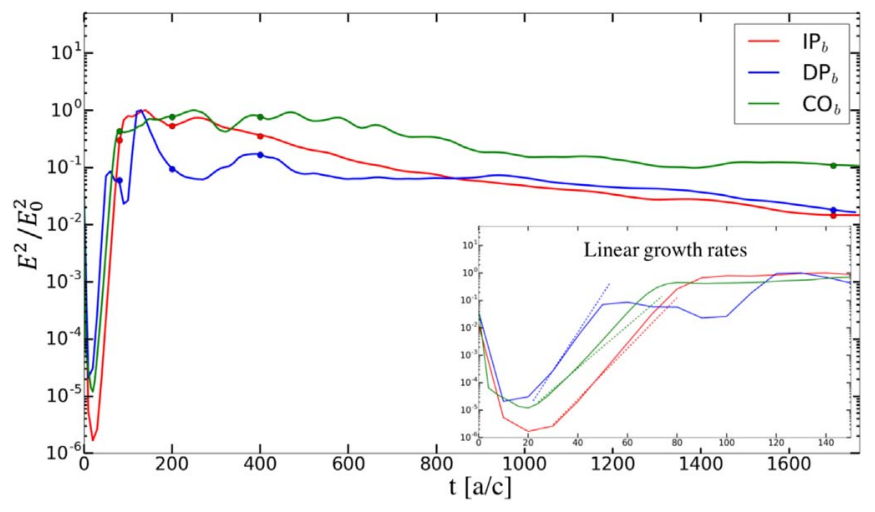

Figure 5. Evolution of the kink mode shown as the electric energy in the three simulated profiles: $\mathrm{IP}_{\mathrm{b}}, \mathrm{DP}_{\mathrm{b}}$, and $\mathrm{CO}_{\mathrm{b}}$. Three phases are evident: (i) linear growth; (ii) mode inverse cascade; and (iii) turbulence phase. The large dots on the three curves, mark the times at which the snapshots in Figures 2-4 are taken. Comparisons to the theoretical linear growth rates of $k_{\max }$ (Equation (3)) are shown as dotted lines plotted over the growth curves of $E^{2}$ in the subplot at the bottom-right corner. In all three models the growth rates are in good agreement with the theoretical predictions.

dissipation. Thus, out of the remaining energy about half may still dissipate, implying that the system is still not in a stable state. This result is consistent with the fact that the dissipation in the DP case did not saturate by the end of the simulation. The obtained value of $\alpha R_{j}=3.66$ is consistent with the range $3.176 \leqslant \alpha R_{j} \leqslant 3.832$ for marginal instability. This also indicates that the dissipation did not finish evolving to its minimal energy state.

In the $\mathrm{CO}$ case, the final magnetic field configuration fits a Taylor profile across most of $R_{j}$ (Figure 9), however with $\alpha R_{J} \ll 3.176$. This manifests the fact that $B_{z} \gg B_{\varphi}$ everywhere in the box. By the end of the simulation about $65 \%$ of the toroidal field energy inside $R_{j}$ has been dissipated (Figure 11), which is equivalent to $8 \%$ of the total EM energy. Such a case is inapplicable for the linear stability analysis

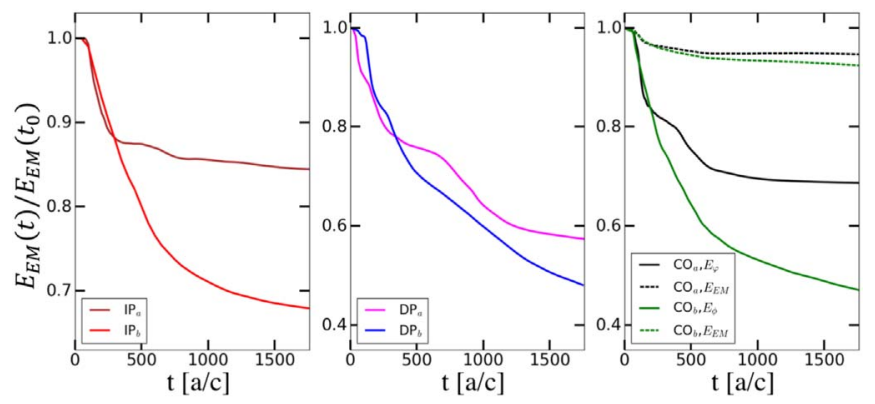

Figure 6. EM energy dissipation in the three profiles studied. In each profile we show the dissipation in the small and big boxes (subindices $a$ and $b$, respectively). We show the total EM energy $\left(\int\left(E^{2}+B^{2}\right) d V\right)$ in the box normalized by the value at $t=0$. For the CO case we show also the total energy without the contribution of $B_{z}, \int\left(E^{2}+B_{\varphi}^{2}\right) d V$ normalized by its initial value as well. The latter is the part that undergoes most of the dissipation in the CO case.

presented in Section 3, as it assumes that the first zero of $J_{0}$ falls inside $R_{j}$. We are therefore unable to estimate how far is the configuration from the minimal energy state. It is noted that the dissipation by this time did not saturate (Figure 6).

\section{Astrophysical Implications}

\subsection{Relativistic Jets}

Kink instability occurs in narrow plasma columns dominated by a toroidal field. Among the systems that may be affected by such process are collimated relativistic jets. A relativistic jet propagating in a medium forms an over-pressurized cocoon around it, which applies pressure on the jet and collimates it. At the launching point the jet pressure is much larger than that of the cocoon and the jet expands radially with an initial opening angle $\theta_{0}$. As the jet material expands and accelerates, its pressure drops faster than the pressure of the surroundings until it becomes equal to the cocoon pressure at $z_{\text {coll }}$ and the jet 

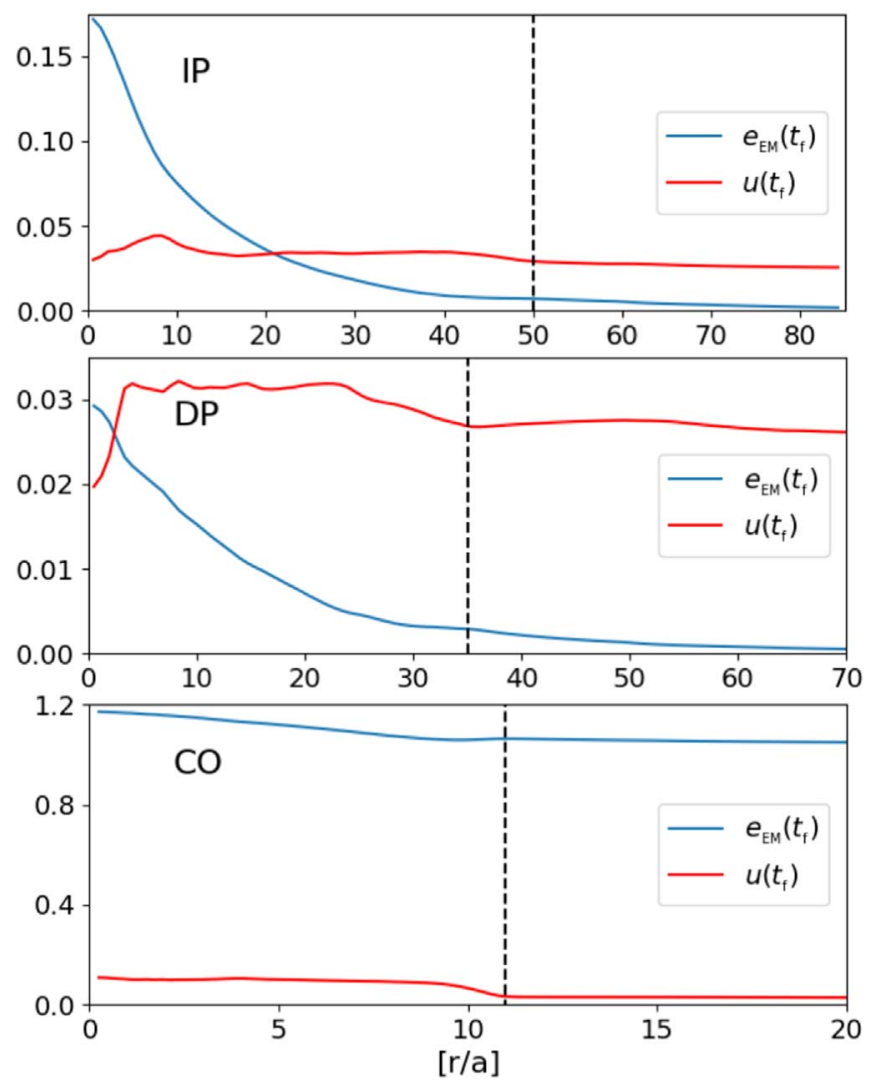

Figure 7. $\mathrm{EM}$ energy density, $e_{\mathrm{EM}}=\frac{1}{8 \pi}\left(E^{2}+B^{2}\right) \simeq \frac{B^{2}}{8 \pi}$ and the thermal energy density, $u=T_{00}-\rho \Gamma^{2} c^{2} \simeq 3 p$ averaged over $z$ and $\varphi$. Shown are the distributions at times $t_{f}$ from simulations $\mathrm{IP}_{\mathrm{b}}$ (top), $\mathrm{DP}_{\mathrm{b}}$ (middle), and $\mathrm{CO}_{\mathrm{b}}$ (bottom). The dashed vertical lines depict the dissipation radius, $R_{j}$, in the three profiles. Though the ratio of magnetic to thermal energy density varies significantly between the profiles, the pressure profile inside $R_{j}$ is flat, implying that the plasma is dominated by the EM forces and is largely at a force-free state.

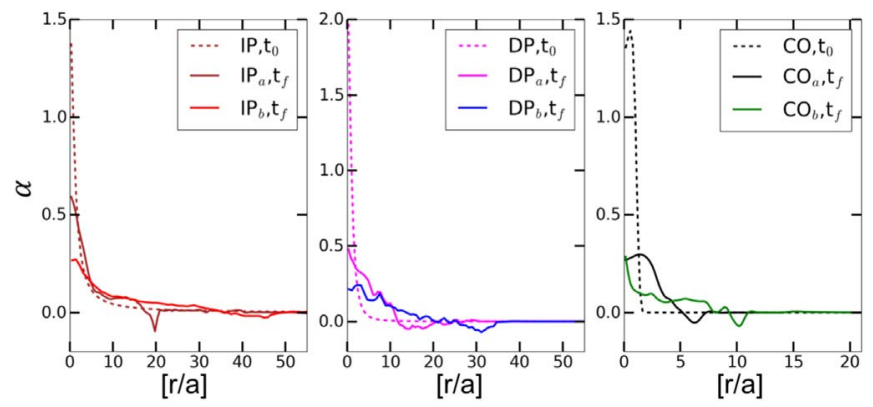

Figure 8. Distribution of $\alpha$, averaged over $z$ and $\phi$ at different times for the three studied profiles. Shown are the profiles at time $t=0$ and at $t=t_{f}$ in the small and the big boxes (subindices $a$ and $b$, respectively). In all cases the distributions in the large boxes are flatter, indicating that the systems are close to a minimal energy state.

becomes collimated. Figure 12 illustrates the geometry of the collimated jet.

If before the jet plasma reaches $z_{\text {coll }}$ it crosses a fastmagnetosonic surface, the collimation is accompanied by the formation of a weak shock. Downstream of the shock the fluid is sub fast-magnetosonic, and decelerates as it expands until it
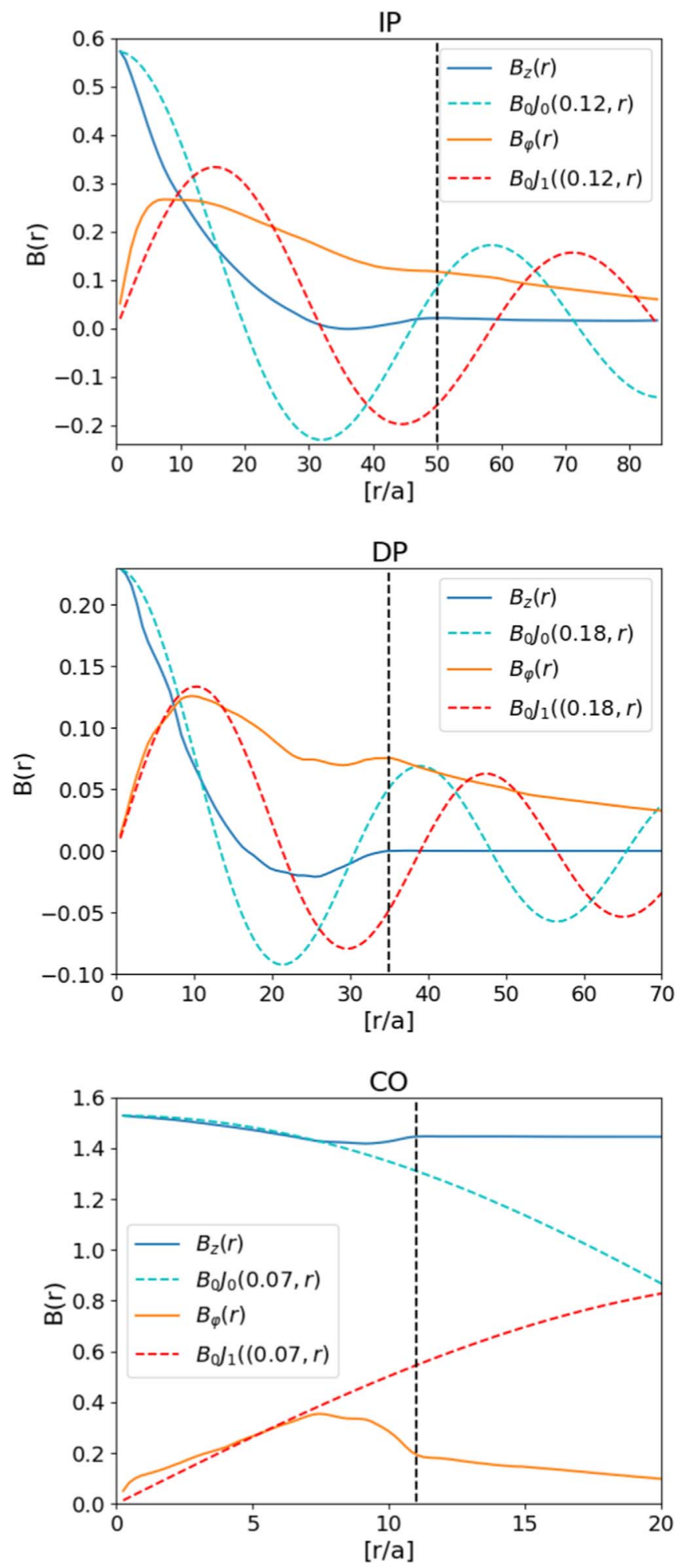

Figure 9. Fitting $B_{0} J_{0}(\alpha r)$ and $B_{0} J_{1}(\alpha r)$ to $B_{z}(r)$ and $B_{\varphi}(r)$ profiles at the end of each simulation. The best-fitted $\alpha$ values are $0.18,0.07$, and $0.12[1 / a]$ for the DP coronal and IP profiles, respectively. The black dashed lines mark the edges of the dissipated regions, $R_{j}$.

reaches $\gamma \beta \sim 1$ at $z_{\text {coll }}$. Conservation of magnetic flux implies that the magnetic field value at the collimation point is

$$
B_{p, c}=B_{L}\left(\frac{R_{L}}{R_{\text {coll }}}\right)^{2} ; B_{\phi, c}=B_{L} \frac{R_{L}}{R_{\text {coll }}},
$$



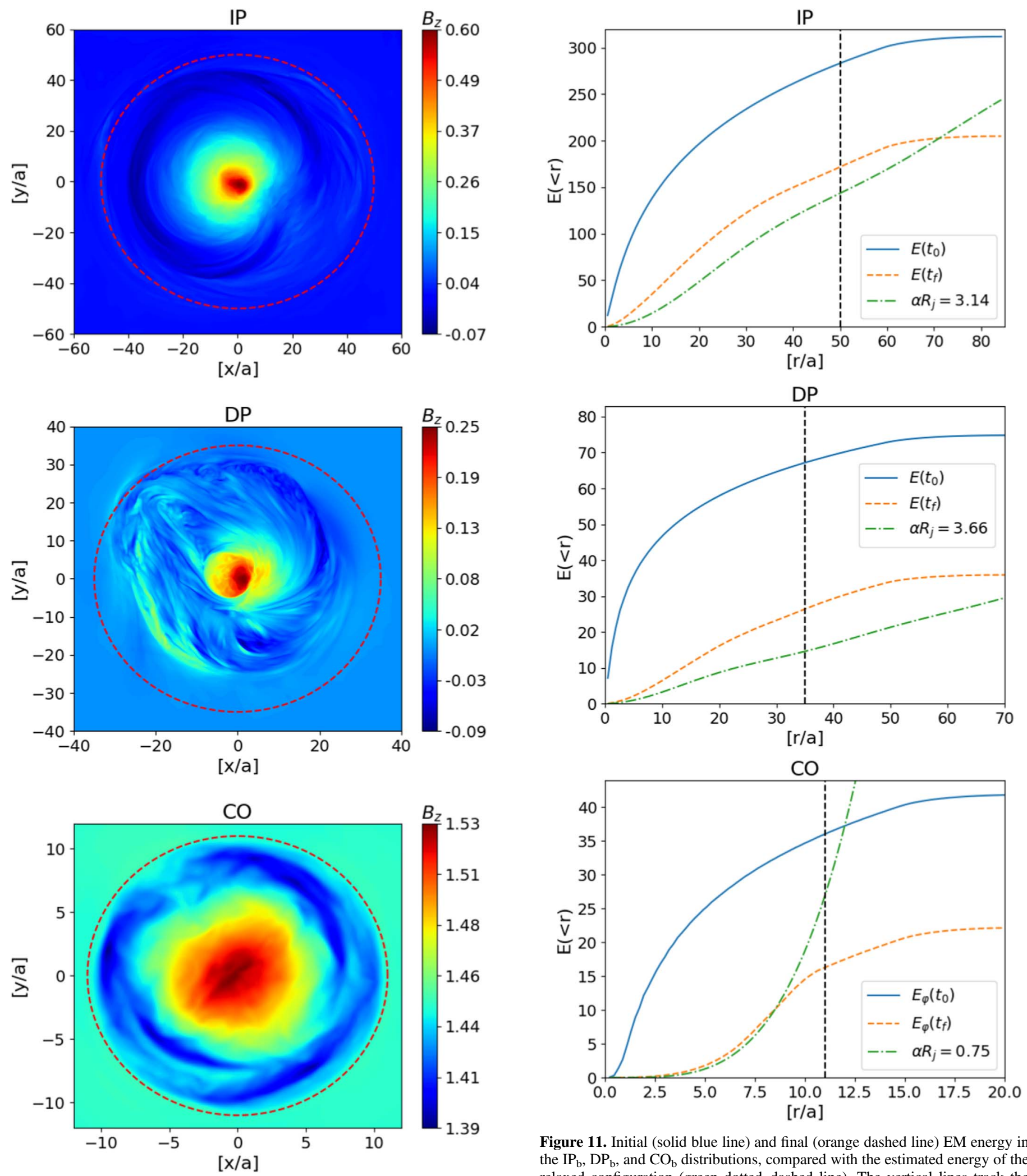

Figure 11. Initial (solid blue line) and final (orange dashed line) EM energy in the $\mathrm{IP}_{\mathrm{b}}, \mathrm{DP}_{\mathrm{b}}$, and $\mathrm{CO}_{\mathrm{b}}$ distributions, compared with the estimated energy of the relaxed configuration (green dotted-dashed line). The vertical lines track the radii of the dissipated regions. In the case of $\mathrm{CO}$ configuration we show only the energy of $B_{\varphi}$.

cross-sectional cut in the middle of the computational box. Field reversals are evident in the IP and DP cases but not in the CO case. The red dashed line marks $R_{j}$ in each case.

where $R_{\text {coll }}=z_{\text {coll }} \theta_{0}$ is the jet cylindrical radius at $z_{\text {coll }}, R_{L}$ is the light cylinder radius, $B_{L}$ is the magnetic field on $R_{L}$, and we assume a conical expansion up to $z_{\text {coll }}$. If $R_{\text {coll }} \gg R_{L}$, the hoop

stress of the toroidal component overcomes the magnetic pressure gradient and the flow converges to the axis (Lyubarsky 2009). As it contracts, the flow accelerates like 


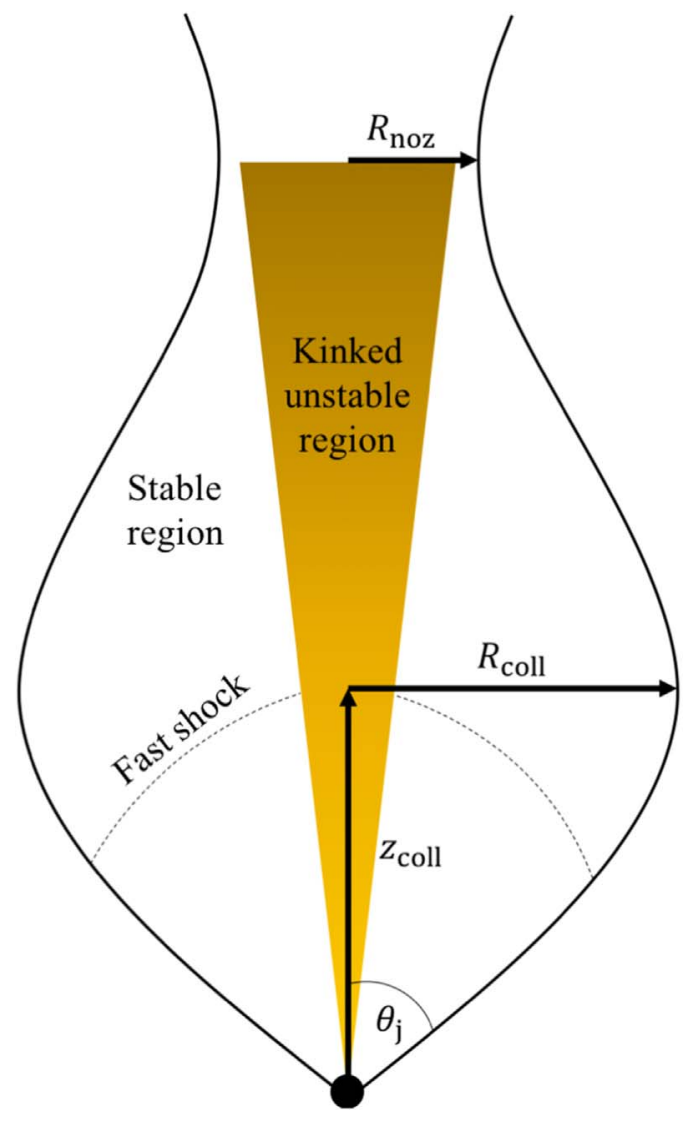

Figure 12. A sketch of the collimation region of a highly magnetized relativistic jet. The jet is conical up to $z \simeq z_{\text {coll }}$, where its pressure becomes equal to the pressure of the surrounding medium. Above this point the collimated flow is affected by the contracting "hoop stress" of $B_{\varphi}$ and converges to the axis. Though the converging flow is in strong causal contact it remains stable for kink due to its fast acceleration. At the center of the jet there is a region where the plasma remains sub-superfast and maintains strong lateral causal contact (yellow region). The flow remains in contact with the nozzle and is unable to accelerate efficiently. It can therefore become kink unstable. If the cross section of the unstable region is comparable to $R_{\text {noz }}$ at the nozzle, the converging plasma from the outer, stable parts will interact with it, get shocked, and become kink unstable as well, resulting in an overall dissipation of the jet EM energy.

(Sobacchi \& Lyubarsky 2018)

$$
\gamma=R_{\text {coll }} / r
$$

where $r$ is the local cylindrical radius, and the magnetic field components in the comoving frame maintain

$$
\begin{aligned}
& b_{p} \simeq B_{p, c}\left(\frac{R_{\text {coll }}}{R}\right)^{2} \\
& b_{\phi} \simeq B_{\phi, c} \frac{R_{\text {coll }}}{R \gamma},
\end{aligned}
$$

where lower case is used to describe comoving quantities and upper case for lab frame values. The convergence to the axis stops when $b_{p} \simeq b_{\phi}$, which by substituting Equations (19) and (20) translates to a nozzle cross-sectional radius of

$$
R_{\mathrm{noz}} \simeq \sqrt{R_{L} R_{\mathrm{coll}}} \simeq \sqrt{R_{L} z_{\mathrm{coll}} \theta_{j}}
$$

The acceleration of the jet material below the collimation point causes it to lose causal contact with the axis, making the plasma stable to global instabilities such as the kink. As it passes the collimation point and begins to contract, the flow regains causal contact and instabilities can grow. However, the fast acceleration of the flow on the converging flow lines does not allow enough time for the instability to grow in the proper frame, and so the instability grows only linearly with $1 / r$ (Sobacchi \& Lyubarsky 2018). Thus, ideally kink instability is unlikely to produce strong dissipation in the flow, both below and above the collimation point (Barniol Duran et al. 2017).

Close to the axis there are field lines with small opening angles, which never lose lateral causal contact. The flow in this region remains in contact with the nozzle and is unable to accelerate efficiently below $z_{\text {coll }}$. The unstable region is marked in Figure 12 as a yellow triangle. The evolution of the instability in this case is expected to be close to that of a stationary plasma column similar to the ones studied here (Sobacchi et al. 2017). Bromberg \& Tchekhovskoy (2016) obtained a relation for the opening angle of the field lines in the unstable region, under the requirement that the plasma on the field lines will be sub fast-magnetosonic and maintain lateral strong causal contact:

$$
\theta_{\text {diss }}=\left\{\begin{array}{l}
\sqrt{\frac{R_{\mathrm{L}}}{z_{\text {coll }}}}, \quad z_{\text {coll }}<R_{\mathrm{L}} \sigma_{0}^{2 / 3}, \\
\frac{R_{\mathrm{L}}}{z_{\text {coll }}} \sigma_{0}^{1 / 3}, \quad z_{\text {coll }} \geqslant R_{\mathrm{L}} \sigma_{0}^{2 / 3}
\end{array} .\right.
$$

At opening angles $<\theta_{\text {diss }}$ the flow is unstable to kink and dissipates its magnetic energy. When it reaches the nozzle it forms an inner core of dissipated plasma. Since the dissipation predominantly destroys the toroidal field, the core plasma will be less affected by the hoop stress and is not expected to converge to the axis like the outer jet part. Therefore its opening angle cannot be smaller than $\theta_{\text {diss. }}$. In fact, it can even be larger due to interaction with material that moves on outer field lines, converges onto the dissipated core, get shocked, and become kink unstable. If the lateral size of the kinked unstable core at the nozzle is comparable to the width of the nozzle (Equation (22)), most of the plasma passing through the nozzle will get shocked and dissipate its energy. Estimating the radius of the kinked unstable core as $R_{\text {diss }} \simeq \theta_{\text {diss }} z_{\text {coll }}$ and requiring that at the nozzle $R_{\text {diss }} \gtrsim R_{\text {noz }}$ we obtain a critical collimation altitude

$$
z_{\text {crit }} \leqslant R_{L} \sigma_{0}^{2 / 3} \theta_{j}^{-1}
$$

below which the entire jet material will undergo efficient magnetic dissipation at the nozzle. If $z_{\text {coll }} \gg z_{\text {crit }}$, the crosssectional radius of the kinked unstable core becomes much smaller than that of the nozzle and most of the jet plasma will pass through the nozzle without interacting with the kinked core and thus may not dissipate its magnetic energy (see e.g., Barniol Duran et al. 2017).

In GRBs at the time the jet breaks out of the star, $z_{\text {coll }}$ $\lesssim R_{*} / 10$, where $R_{*} \simeq 10^{11} \mathrm{~cm}$ is the stellar radius of the host star. The critical nozzle altitude is

$$
z_{\text {crit }} \simeq 10^{10} R_{7} \sigma_{3}^{2 / 3} \theta_{-1}^{-1} \mathrm{~cm} .
$$

After the breakout, the cocoon surrounding the jet loses pressure through a rarefaction wave that propagates from the surface inwards toward the collimation point. The wave reaches $z_{\text {coll }}$ a few tens of seconds after the breakout and reduces the cocoon pressure there. As a result the collimation becomes ineffective, leading to a wider nozzle, which could stop the 
magnetic dissipation. This raises an interesting possibility that the observed duration of the prompt GRB emission might be connected with the efficient dissipation of the jet's magnetic energy at the collimation nozzle. Further study of the time evolving conditions at the nozzle before and after the breakout is required to validate this scenario.

\subsection{Accretion Disks}

Kink instability can also play an important role in dissipating magnetic energy of twisted loops above accretion disks. Geometrically thin accretion disks near active galactic nuclei can support highly magnetized coronae consisting of smallscale magnetic flux tubes (e.g., Galeev et al. 1979), which is thought to power a bright compact X-ray source in a "lamppost" or "extended coronae" models (Parfrey et al. 2015; Yuan et al. 2019). Flux tubes are twisted by the disk differential rotation and may eventually become kink unstable under the strong confinement from the neighboring vertical field (Yuan et al. 2019). This situation closely resembles the coronal configuration tested in Bodo et al. (2013) and in this work. Our results imply that the energy of the toroidalmagnetic field stored in the loop gets quickly converted into plasma thermal energy via dissipation in multiple current sheets. As we show, the large-scale current sheets break into turbulence that can further dissipate the magnetic energy in a significant fraction of the volume of the disk's corona. Similar flares powered by reconnection in kink unstable overtwisted magnetic loops can happen in magnetospheres of magnetars (Beloborodov 2009). Simulations of reconnection driven by kink instability in high-sigma plasma in the loop geometrical configuration will be necessary to quantify the dissipation rate and magnetic energy release.

\section{Discussion and Conclusions}

We show that kink instability growing in relativistic magnetized plasma columns can lead to efficient dissipation of magnetic field that continues until the configuration relaxes to a state with minimal free energy. In the case of nonrotating columns, this state corresponds to a force-free Taylor state (Taylor 1974). This, however, requires a global process that efficiently dissipates the magnetic field energy. In this work we show that the mechanism is driven reconnection, induced by the continuous growth of the kink instability (in the nonlinear stage) followed by coalescence of the kink mode to lower longitudinal wave-number modes.

We identify three stages of the dissipation that correspond to three episodes in the evolution of the instability. (i) Nonlinear stage: at the end of the linear stage, the growth of the kink mode saturates. The growing mode shears the magnetic field configuration inwards to the wave front. The twisted column presses against the non-twisted plasma outside forming a prominent helical current sheet at the wave front. (ii) Mode merging: as the instability continues to grow the kink lobes, which expand in the longitudinal direction as well, touch each other and begin to merge. The merging process forces the magnetic fields to reconnect at a high rate. It also drives smallscale turbulence, which breaks the current sheet, mixes the magnetic fields, and helps bring the plasma to the Taylor equilibrium state. (iii) Relaxation: the growth of the kink instability relaxes once the kink mode reaches the lowest $k$ allowed in the box. The small-scale turbulence continues to dissipate the energy contained in them at a slower rate until the configuration becomes fully relaxed.

The dissipation rate as well as the total energy dissipated depend on the magnetic field configuration. Configurations in which the pitch is rising have a resonant surface that tends to regulate the dissipation. The mode coalescence is gradual and the wave number decreases progressively to the lowest value. Configurations in which the pitch is decreasing do not have a resonant surface. They are less stable and experience a more instantaneous coalescence of the kink mode into the minimal wave number allowed in the box. As a result, the dissipation rate is higher and the total amount of dissipated energy is larger. In our setups, about $40 \%$ of the EM energy was dissipated by the end of the simulation in the IP case, compared with $60 \%$ of dissipated energy in the DP case (see Figure 11). We estimated through linear stability consideration that the available energy for dissipation in these two cases is $50 \%$ and $75 \%$ for the IP and DP cases, respectively. The coronal configuration tested here behaves similar to the IP case and seems to dissipate a similar fraction of the toroidal field energy.

We find a toroidal field dissipation rate $d U_{B \phi} / d t \approx$ $-0.1 U_{B \phi} / \tau$, where

$$
\tau \approx 20 \pi P_{0} / v_{\mathrm{A}}
$$

is the growth time of the linear kink instability. This rate is qualitatively consistent with the measured sideways motion velocity of $0.1 c$, which drives the reconnection in the current sheet at the boundary of the kinked column.

Our simulations show that the relaxation criterion for kinkinduced dissipation is a minimal energy state, close to the Taylor state. Although thermal pressure becomes important during the dissipation, we observe it to flatten out during the relaxation, resulting in a force-free configuration. We therefore conclude that the thermal pressure likely does not play a role in stabilizing the system. In the cases of monotonic pitch profiles (IP and DP) where internal kink is evolving, the twisting of $B_{z}$ results in a reversed field at the outer parts of the dissipated region. This allows the system to relax into a Taylor state, with parameters defined by the conditions of marginal stability. We stress that the ideal value was obtained for $m=-1$ kink modes, while the final stage of the evolution is dominated by turbulent dissipation. The connection to the linear stability criterion likely comes from the fact that the energy in the turbulence originates in the inverse cascade of the kink mode, thus they share the same energy reservoir. In the coronal case the strong $B_{z}$ in the ambient medium prevents a field reversal. The topology of the field does not change much and the minimal energy state is close to the initial one. During the evolution of the kink instability, the radius of the kinked unstable core is slowly increasing. The core pushes against the magnetic field in the medium, resulting in the growth of instabilities at the boundary, which mixes external matter into the core. The origin and outcome of such mixing need to be further studied with numerical simulations.

To reach a minimal energy state, the kink mode has to go through enough merger episodes as it inverse cascades to the lowest wave number allowed in the box, in order to pump enough energy into the turbulence. This requires a large enough box that will allow for the growth of a kink mode with a large $n$. If the computational box is too small, the kink instability relaxes before the plasma has time to reach the Taylor state, and the final magnetic energy is higher. Such a situation is seen 
in the small box simulation of the increasing pitch $\left(\mathrm{IP}_{\mathrm{a}}\right)$. Turbulence continues to dissipate energy even after the kink instability relaxes, however the dissipation rate is significantly smaller compared to the mode inverse cascade stages.

Last, we obtained through analytic considerations the conditions in which kink instability can play a significant role in dissipating the magnetic energy in relativistic collimated jets. These conditions need to be verified in global numerical simulations we intend to perform in future work.

\subsection{Implications for Particle Acceleration}

In MHD simulations without explicit resistivity the dissipation happens on the grid scale. The hope is that with sufficient numerical resolution separation of the dissipation scale, e.g., cell size and the column size are sufficiently large to represent a realistic astrophysical system. To prove this, we checked that our dissipation rates and dissipated energy fractions are converged with numerical resolution (see Appendix B for convergence tests).

To move further, particle-in-cell (PIC) kinetic plasma simulations can provide insight into how the magnetic dissipation in kink instability results in nonthermal particle acceleration. In Davelaar et al. (2019) we perform PIC simulations for the setups studied in this work. We show that if the jet size is sufficiently large, the kink instability grows at a rate very similar to the ideal MHD instability. We also show that current sheets, which form in the nonlinear phase of the instability accelerate particles in the initially cold plasma to a nonthermal distribution. The current sheets later break into small-scale turbulence, similar to what we observe in the MHD simulations and continues to dissipate magnetic energy into heat. Future PIC simulations with larger scale separation will allow to better probe the interplay between acceleration in turbulence and reconnection (e.g., Alves et al. 2018, 2019; Zhdankin et al. 2018, 2019). A complementary approach for achieving greater scale separation between the jet size and the dissipation scale might be to perform large-scale resistive MHD simulations with a resistivity prescription motivated by PIC simulations and trace particles through these simulations (Ripperda et al. 2017).

We would like to thank the Flatiron Institute for its generous hospitality and the 14th Summer School of Modern Astrophysics at MIPT, where part of this work was done. We would also like to thank A. Bhattacharjee, M. Medvedev, B. Ripperda, and A. Tchekhovskoy for stimulating and insightful discussions and useful comments. The study was partially funded by an ISF grant 1657/18 (O.B. and C..S.), by an ISF (I-CORE) grant 1829/12 (O.B. and C.S.), and by a BSF grant 2018312 (O.B. and S. P.). J.D. is supported by the ERC Synergy Grant "BlackHoleCam-Imaging the Event Horizon of Black Holes" (grant 610058, Goddi et al. 2017). The Flatiron Institute is supported by the Simons Foundation.

Software: PLUTO (Mignone et al. 2007, 2012), python (Oliphant 2007; Millman \& Aivazis 2011), scipy (Jones et al. 2001), numpy (van der Walt et al. 2011), matplotlib (Hunter 2007), VisIt (Childs et al. 2005).

\section{Appendix A}

A configuration of an axially symmetric magnetic field with vanishing $B_{r}$ on the boundary evolves while conserving total helicity and total magnetic flux. The helicity of such configuration can be described as

$$
H(R)=2 \pi L\left[2 \int_{0}^{R} \frac{\Psi\left(r^{\prime}\right)}{2 \pi} \frac{2 I\left(r^{\prime}\right)}{r^{\prime}} d r^{\prime}+\left.\left(A_{z} \frac{\Psi}{2 \pi}\right)\right|_{0} ^{R}\right]
$$

where $\Psi(r)$ is the magnetic flux within radius $r$ defined as

$$
\Psi(r)=2 \pi \int_{0}^{r} B_{z} r^{\prime} d r^{\prime},
$$

and $I(r)$ is the current within that radius. Taking a gauge $A_{z}(R)=0$, the second term vanishes and we are left with the first, which we identify as

$$
K(R) \equiv 2 \int_{0}^{R} \frac{\Psi\left(r^{\prime}\right)}{2 \pi} \frac{2 I\left(r^{\prime}\right)}{r^{\prime}} d r^{\prime}
$$

$K\left(R_{j}\right)$ is largely conserved throughout the evolution of the system.

If the system evolves to a Taylor state, its magnetic field components can be described by a pair of Bessel functions of the first kind:

$$
\begin{aligned}
& B_{z}=B_{0} J_{0}(r \alpha) \\
& B_{\phi}=B_{0} J_{1}(r \alpha) .
\end{aligned}
$$

In this case the vector potential can be expressed as $A_{\varphi}(r)=B_{\varphi}(r) / \alpha, A_{z}=B_{z}(r) / \alpha$ resulting in an helicity

$$
\begin{aligned}
H & =2 \pi L \int_{0}^{R_{j}}(\boldsymbol{A} \cdot \boldsymbol{B}) r d r \\
& =\frac{2 \pi L}{\alpha} B_{0}^{2} \int_{0}^{R_{j}}\left[J_{0}(\alpha r)^{2}+J_{1}(\alpha r)^{2}\right] r d r .
\end{aligned}
$$

This helicity maintains a gauge, $A_{z}\left(R_{j}\right)=\frac{B_{0}}{\alpha} J_{0}\left(\alpha R_{j}\right)$. Substituting that into Equation (27), we can express the helicity in terms of $K$ :

$$
H=2 \pi L\left[K\left(R_{j}\right)+\frac{B_{0}^{2}}{\alpha} J_{0}\left(\alpha R_{j}\right) \int_{0}^{R_{j}} J_{0}(\alpha r) r d r\right],
$$

with

$$
\begin{aligned}
K= & \frac{B_{0}^{2}}{\alpha}\left(\int_{0}^{R_{j}}\left[J_{0}(\alpha r)^{2}+J_{1}(\alpha r)^{2}\right] r d r\right. \\
& \left.-J_{0}(\alpha r) \int_{0}^{R_{j}} J_{0}(\alpha r) r d r\right)
\end{aligned}
$$

The total flux maintains

$$
\Psi=2 \pi B_{0} \int_{0}^{R_{j}} J_{0}(\alpha r) r d r .
$$

To calculate the total energy in the relaxed state we need to obtain thee parameters $B_{0}, \alpha$, and $R_{j}$, thus an additional constraint is required in order to close the equations. For example, we can take the constraint of $\alpha R_{j}$ of the minimal energy configuration obtained from the linear stability analysis by (Voslamber \& Callebaut 1962):

$$
\alpha R_{j}=3.176
$$



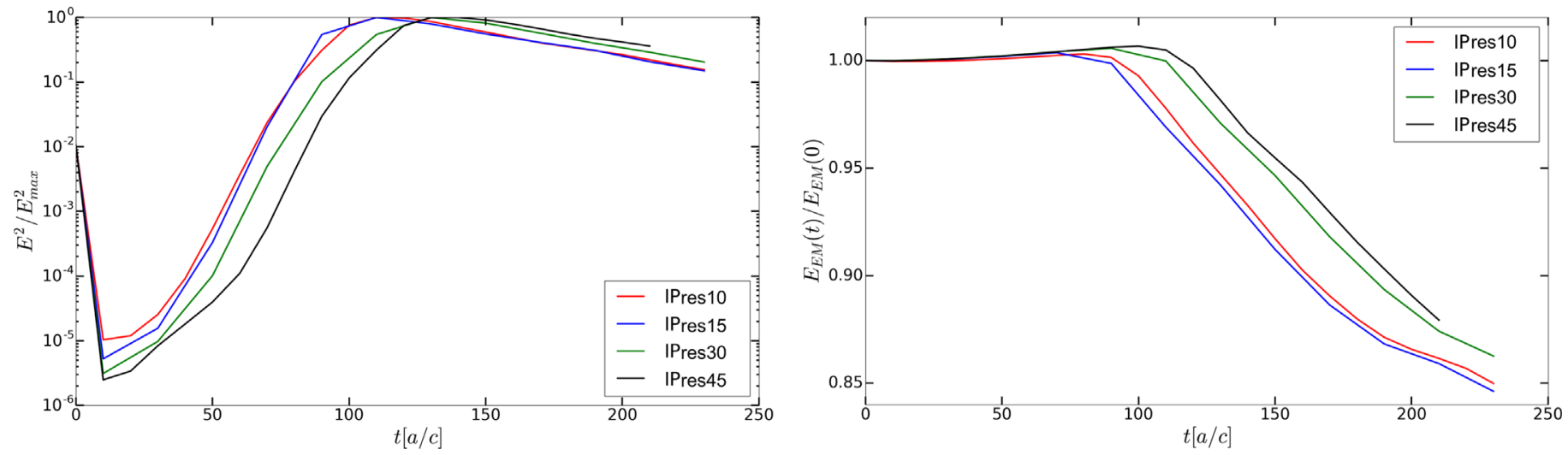

Figure 13. Growth rates of the kink instability, represented by the value of $E^{2}$ (left) and the EM energy dissipation rates (right) in the IP configuration. Simulations were performed in a box of size $40 \times 40 \times 20 a$ with a resolution of $10,15,30$, and 45 computational cells per unit length $a$.

to get the three unknowns

$$
\begin{aligned}
B_{0} & =\left(\frac{K}{\tilde{\Upsilon}}\right)^{2}\left(\frac{2 \pi}{\Psi} J_{1}(3.176) \cdot 3.176\right)^{3} \\
\alpha & =\frac{K}{\tilde{\Upsilon}}\left(\frac{2 \pi}{\Psi} J_{1}(3.176) \cdot 3.176\right)^{2} \\
R_{j} & =\frac{3.176}{\alpha},
\end{aligned}
$$

with

$$
\begin{aligned}
\tilde{\Upsilon} & =\int_{0}^{3.176}\left[J_{0}(\xi)^{2}+J_{1}(\xi)^{2}\right] \xi d \xi \\
& -J_{0}(3.176) J_{1}(3.176) \cdot 3.176,
\end{aligned}
$$

a constant obtained from Equation (34), and we used the relation $\int_{0}^{R} J_{0}(r) r d r=R J_{1}(R)$. Alternatively, we can use the $R_{j}$ of the simulations to extract $B_{0}$ and $\alpha$ from Equations (34) and (35). This will define the properties of the Taylor state that corresponds to $R_{j}$ and conserved the helicity and magnetic flux in the box up to $R_{j}$. We can then compare $\alpha R_{j}$ to the expected value from linear stability analysis and estimate how close is the distribution to a relaxed, minimal energy state.

\section{Appendix B}

Reconnection in ideal MHD simulations is triggered by numerical resistivity. In order to verify that the actual value of the resistivity does not affect the physics of the dissipation process, we examined the dissipation during the nonlinear stage of the kink instability for different numerical resolution. Here we report the tests performed for the IP configuration. We set up the same initial and boundary conditions as in the production runs and compared the evolution for resolutions of $10,15,30$, and 45 computational cells per unit length $a$. Figure 13 shows the linear growth rates of the kink instability (left) and the associated EM energy dissipation rates (right). The simulations were made in a box smaller than that used in our production runs, to allow for manageable run times at high resolutions $(40 a \times 40 a \times 20 a)$. The growth rates and the dissipation rates at all four resolutions are almost identical. There is a spread in the peak time of the electric field of $\sim 20 a$ / $c$, which corresponds to a similar delay time in the onset of the linear growth. This spread leads to a $2 \%$ difference in the dissipated energy at a time of $200 a / c$. We have chosen a resolution of 15 computational cells per unit length $a$ for our production runs. This allows us to run the larger box simulations at a reasonable time and to capture the right physics of the dissipation process.

\section{ORCID iDs}

Chandra B. Singh (i) https://orcid.org/0000-0002-7782-5719 Alexander A. Philippov (i) https://orcid.org/0000-00017801-0362

\section{References}

Alves, E. P., Zrake, J., \& Fiuza, F. 2018, PhRvL, 121, 245101

Alves, E. P., Zrake, J., \& Fiuza, F. 2019, PhPl, 26, 072105

Anjiri, M., Mignone, A., Bodo, G., \& Rossi, P. 2014, MNRAS, 442, 2228

Appl, S. 1996, A\&A, 314, 995

Appl, S., Lery, T., \& Baty, H. 2000, A\&A, 355, 818

Barniol Duran, R., Tchekhovskoy, A., \& Giannios, D. 2017, MNRAS, 469, 4957

Begelman, M. C. 1998, ApJ, 493, 291

Beloborodov, A. M. 2009, ApJ, 703, 1044

Blandford, R. D., \& Znajek, R. L. 1977, MNRAS, 179, 433

Bodo, G., Mamatsashvili, G., Rossi, P., \& Mignone, A. 2013, MNRAS, 434, 3030

Bromberg, O., \& Tchekhovskoy, A. 2016, MNRAS, 456, 1739

Browning, P. K., Gerrard, C., Hood, A. W., Kevis, R., \& van der Linden, R. A. M. 2008, A\&A, 485, 837

Childs, H., Brugger, E. S., Bonnell, K. S., et al. 2005, in Proc. IEEE Visualization 2005 Conf. (Piscataway, NJ: IEEE), 191, doi:10.1109/VISUAL.2005.1532795

Davelaar, J., Philippov, A. A., Bromberg, O., \& Singh, C. B. 2019, PhRvL, submitted

Freidberg, J. P., \& Haas, F. A. 1973, PhFl, 16, 1909

Galeev, A. A., Rosner, R., \& Vaiana, G. S. 1979, ApJ, 229, 318

Goddi, C., Falcke, H., Kramer, M., et al. 2017, IJMPD, 26, 1730001

Gordovskyy, M., \& Browning, P. K. 2011, ApJ, 729, 101

Hawley, J. F., Fendt, C., Hardcastle, M., Nokhrina, E., \& Tchekhovskoy, A. 2015, SSRv, 191, 441

Hood, A. W., \& Priest, E. R. 1979, SoPh, 64, 303

Hunter, J. D. 2007, CSE, 9, 90

Istomin, Y. N., \& Pariev, V. I. 1996, MNRAS, 281, 1

Jones, E., Oliphant, T., \& Peterson, P. 2001, SciPy: Open Source Scientific Tools for Python, http://www.scipy.org/

Kadomtsev, B. B. 1975, SvJPP, 1, 710

Komissarov, S. S. 2001, MNRAS, 326, L41

Kruskal, M., \& Tuck, J. L. 1958, RSPSA, 245, 222

Lery, T., Baty, H., \& Appl, S. 2000, A\&A, 355, 1201

Lyubarskii, Y. E. 1999, MNRAS, 308, 1006

Lyubarsky, Y. 2009, ApJ, 698, 1570

Mignone, A., Bodo, G., Massaglia, S., et al. 2007, ApJS, 170, 228

Mignone, A., Rossi, P., Bodo, G., Ferrari, A., \& Massaglia, S. 2010, MNRAS, 402, 7

Mignone, A., Striani, E., Tavani, M., \& Ferrari, A. 2013, MNRAS, 436, 1102

Mignone, A., Zanni, C., Tzeferacos, P., et al. 2012, ApJS, 198, 7 
Millman, K. J., \& Aivazis, M. 2011, CSE, 13, 9

Mizuno, Y., Lyubarsky, Y., Nishikawa, K.-I., \& Hardee, P. E. 2009, ApJ, 700,684

Mizuno, Y., Lyubarsky, Y., Nishikawa, K.-I., \& Hardee, P. E. 2012, ApJ, 757,16

Oliphant, T. E. 2007, CSE, 9, 10

Parfrey, K., Beloborodov, A. M., \& Hui, L. 2013, ApJ, 774, 92

Parfrey, K., Giannios, D., \& Beloborodov, A. M. 2015, MNRAS, 446, L61

Ripperda, B., Porth, O., Xia, C., \& Keppens, R. 2017, MNRAS, 471, 3465

Rosenbluth, M. N., Dagazian, R. Y., \& Rutherford, P. H. 1973, PhFl, 16, 1894

Shafranov, V. D. 1956, At. Energ., 5, 38

Sobacchi, E., \& Lyubarsky, Y. E. 2018, MNRAS, 480, 4948
Sobacchi, E., Lyubarsky, Y. E., \& Sormani, M. C. 2017, MNRAS, 468, 4635

Taylor, J. B. 1974, PhRvL, 33, 1139

Taylor, J. B. 1986, RvMP, 58, 741

Taylor, J. B. 2000, PhPl, 7, 1623

van der Walt, S., Colbert, S. C., \& Varoquaux, G. 2011, CSE, 13, 22

Voslamber, D., \& Callebaut, D. K. 1962, PhRv, 128, 2016

Yuan, Y., Spitkovsky, A., Blandford, R. D., \& Wilkins, D. R. 2019, MNRAS, 487, 4114

Zhdankin, V., Uzdensky, D. A., Werner, G. R., \& Begelman, M. C. 2018, ApJL, 867, L18

Zhdankin, V., Uzdensky, D. A., Werner, G. R., \& Begelman, M. C. 2019, PhRvL, 122, 055101 